\title{
DEVELOPMENT OF OPTIMIZED POINT CLOUDS MERGING METHOD FOR RAPID PROCESSING TO GENERATE EARTHWORK SITE MODEL
}

\author{
Soonwook Kwon ${ }^{1 *}$, Mi Na Lee $^{2}$, Moon Joo Lee ${ }^{3}$, Myungjin Chae ${ }^{4}$, and Junbok Lee ${ }^{5}$ \\ ${ }^{1}$ Associate Professor, Department of Design and Engineering, Department of Civil, Architectural and \\ Environmental System Engineering, Sungkyunkwan University, Suwon, Korea \\ ${ }^{2}$ Department of Civil, Architectural and Environmental System Engineering, \\ Sungkyunkwan University, Suwon, Korea \\ ${ }^{3}$ Department of School of Information \& Communication Engineering, Sungkyunkwan University, Suwon, Korea \\ ${ }^{4}$ Senior Researcher, Korea Institute of Construction Technology, Ilsan, Korea \\ ${ }^{5}$ Associate Professor, Department of Architectural Engineering, Kyung Hee University, Yongin, Korea \\ * Corresponding author (swkwon@skku.edu)
}

\begin{abstract}
As a continuation of the authors' research presented in previous years (ISARC), these authors focus on the processing aspect of the $3 \mathrm{D}$ terrain models acquired from heterogeneous $3 \mathrm{D}$ scan sources. This includes a revision of the authors' ICP-based merging algorithm to achieve better accuracy in the ever-changing shape of an earthwork site with acceptable penalty in speed, and the introduction of a new side-scanning fast laser to obtain accurate local shapes at a reasonably fast time. In conjunction with these issues, the data format for holding the terrain data was also taken into account, for faster processing.
\end{abstract}

Keywords: Registration, 3D Point, Automatic Construction

\section{INTRODUCTION}

Due to the difficulty in improving the productivity in construction sites due to such sites' variety and complexity, automation has become an attractive method of improving productivity. Focusing on one phase of the intelligent excavation system (IES) development process, this study aimed to automate the excavation work using an excavator, and to find the optimal workpath at an earthwork site. In earthworks, automation technology can improve the work quality by preventing safety accidents and other risks. Here, the development of IES targets automation, and accordingly, this study aimed to model and merge the development process at a $3 \mathrm{D}$ virtual space. $3 \mathrm{D}$ virtual modeling is useful in efficiently expressing $3 \mathrm{D}$ models and in creating the appearance of an object in a virtual environment, thus requiring an accurate and appropriate algorithm that can merge the global and local models to produce a world model. This merging algorithm has long been researched on in the fields of robotics and vision studies. ICP (iterative closest point) [1], one of the most well-known and widely used algorithms, is often used by many groups for registration. This paper used not only ICP but also another algorithm to merge the global and local models and to reflect the construction environment (i.e., the site), which is part of nature. ICP is a method that allows one to compare and verify whether all points of one model are identical to those of the other model. Due to the variety of earthwork sites, however, it is difficult to find characteristics that do not change. Already, in the previous stage, the process used GPS to create the global model, and the local model also contains GPS values. If these characteristics are to be used, one can roughly determine where in the global model the local model is located. Compared to the $x, y$ distance, the two models do not show many errors, and the $x, y$ axis rotations are not huge either. Therefore, this paper determines the plane using the $3 \mathrm{D}$ 
point, and rotates the local model before the implementation of ICP using the normal vector. After the movement of the location, it places the local model onto the global model. Then, using the ICP algorithm, correction is made on the $z$ axis rotation.

As such, this study verifies the location information of both the global model, which was acquired from the 3D scanner, and the local model, from the stereoscopic vision, with the GPS measurement, and develops an algorithm to create a world model.

\section{RELATED WORKS}

The recent domestic and foreign research directions for 3D modeling can be categorized into research on the acquisition of effective and varied 3D-form information, and research on developing reliable modeling in a 3D space.

For the acquisition of effective 3D-form information, the research has focused on the fast acquisition of spatial models using the point cloud of laser scanning [4]. Such method will not only allow real-time 3D modeling through terrain laser scanning for modeling in a $3 \mathrm{D}$ space but will also improve the possibility of coming up with an obstacle avoidance system, which will become a required drive for construction automation and will greatly influence various decision-making systems [5].

Image registration is used not only in computer vision but also in construction automation and medical engineering, etc., and is being studied by various research groups. The algorithm that was used in this study was well surveyed in the study conducted by Barbara [6]. ICP, which was used in this study, has also been actively studied in South Korea and abroad. One of the most basic ICP algorithms was first proposed by Besl and McKay [1] in 1992. They used the ICP algorithm to match the freeform with 6 degrees of freedom (6DOF). To raise the speed of the representative registration ICP algorithm, the number of repetition $n$ as well as the data points $N p$ and $N x$ were reduced [7]. Another study on the registration algorithm used the Kernel correlation, which expanded the concept of point sets correlation [8]. These studies used only one type of equipment, a laser scanner, to acquire 3D-form information, and converted and merged the point cloud data of the acquired 3D-form information.

There are no existing data and algorithms, however, for converting and merging 3D-form information in the domestic and foreign construction industries, particularly in earthworks. Therefore, in the following chapter, the data and algorithm required for 3D formation in earthworks are introduced.

\section{ALGORITHM}

\subsection{Setting and Data Collection}

GLOBAL DATA, the point cloud data scanned from the construction site through a 3D scanner, are updated through the MESH GENERATION and delivered to the Task Scanning System. Moreover, the data acquired by scanning the terrain after the excavation work with the stereoscopicvision sensor installed on the excavator during the actual earthwork are collectively called "local model." These data were modeled by considering the specifications of the excavator to improve the compatibility of the task planning system. Here, the local model is included in the global model, and recorded on these two dataset values were the UTM coordinates, which were identical to the terrain information. As a result, the study aimed to determine the terrain of the working site in real time by merging these two datasets.

Shown in Fig. 1 is the earthwork process from the excavation work using an excavator to the task planning system, which allows the optimal workpath.

Once the scan models with different data types coming from each machine are transmitted via a communication protocol, the global and local scan data are stored on the central DB, and to merge the two data in real time, an algorithm-based viewer is required. Further, based on the object data of the world model, task planning is performed to acquire useful information, such as the work allotment and order within the zone, the location of the platform, and the optimal movement path. 
The algorithm that is required to merge the global and local scan data in real time are explained below.

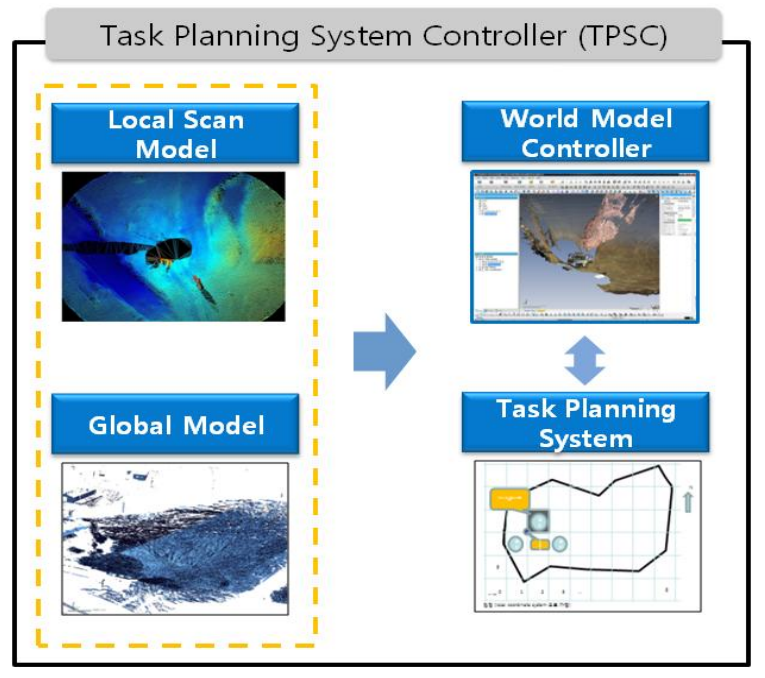

Fig. 1 Data process.

\subsection{Merging Algorithm}

Using GPS, the local data are placed onto the global data. That is, based on the global coordinates, only the $\mathrm{x}$ and $\mathrm{y}$ axes of the measured local data are rotated and placed on top of the global map with a different $z$ value. Shown in Fig. 2 is the merging of the global and local data.

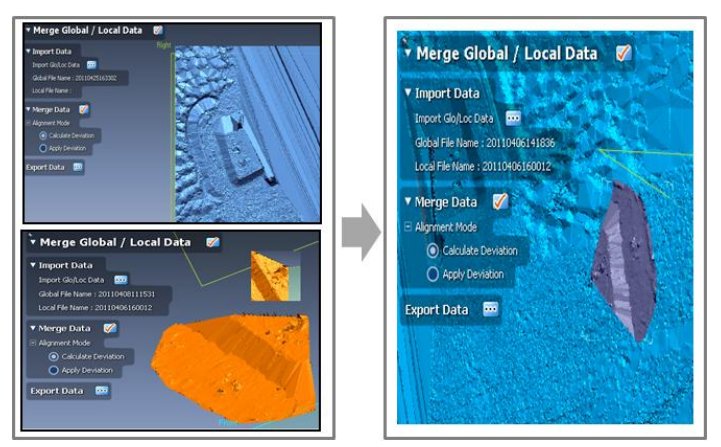

Fig. 2 Merging of the local and global data.

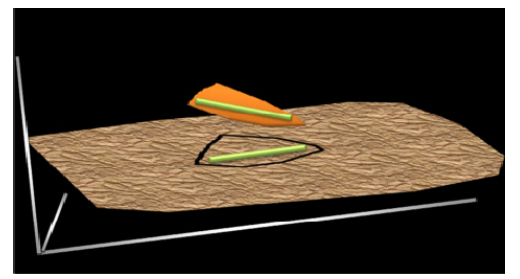

Fig. 3 Local and global models: While the black part of the global model is the place where the local model should be located, the local model (orange) has $\mathrm{z}$ translation, and the $\mathrm{x}, \mathrm{y}$, and $\mathrm{z}$ axes are also rotated.

As shown above, the initial data have $\mathrm{x}$ and $\mathrm{y}$ rotations. Thanks to the GPS values, the location is somewhat reliable. The $\mathrm{z}$ translation, however, as shown above, has a huge difference. As such, the merging of the two data requires the rotation of the $\mathrm{x}$ and $\mathrm{y}$ axes and $\mathrm{z}$ translation.

The two data are merged in the following way. First, the plane on the local data is determined, and the region in the global data that corresponds to the $\mathrm{x}$ and $\mathrm{y}$ axes of the local data is sought. Then the normal vector of the two planes is calculated, which is used as the standard for setting the axis vertical to the normal vector towards the $\mathrm{x}$ axis. Finally, the final axis is calculated using the two axes. After transforming the three axes, the local map will be placed onto the global map, which will complete the first merging process. Second, while the local model is being placed on top of the global model, it will be rotated by the $\mathrm{z}$ axis. To adjust this rotation, ICP is used.

\section{(a) Finding the plane}

First, randomly determine a point in the local area, and take a region whose radius is $R$, with the point as the center, using the spherical equation.

After determining the plane, find the plane on the global region with the same $\mathrm{x}$ and $\mathrm{y}$ coordinates. 


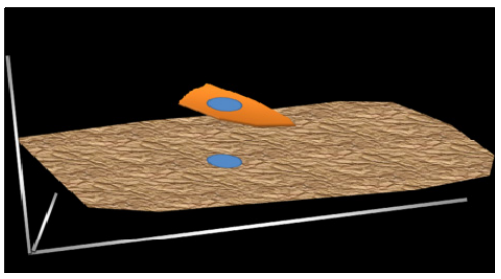

Fig. 4 The detached plane.

Determine the covariance using the three points at the extracted plane, and decompose the covariance into three parts using SVD (singular-value decomposition) [1]. The central matrix corresponds to the eigenvector, which is the normal vector of the plane.

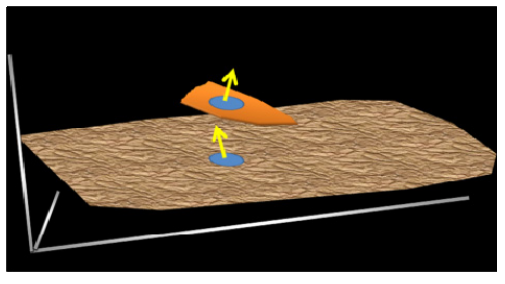

Fig. 5 Normalized plane.

Verify if the taken region is a plane using the normal vector, which can be done by using the normal vector and the major axis.

$$
A x\|B y\| E z+D-0
$$

Acquire more points corresponding to the plane within the fixed region using the plane equation. If it is not a plane, select another spot as a point, and find the plane. This process should be repeated until both regions that are taken satisfy the plane equation.

\section{(b) Transformation-1}

Create a straight line 90 degrees vertical to the a axis from the normal vector, and after translating it by the $\mathrm{x}$ and $\mathrm{y}$ values of the center of the region taken by $(1,0.0)$ vector, project it onto the taken region to create an axis vertical to the normal vector. Another axis, vertical to the two axes, can then result from the cross-production of the two axes. Using the two regions, three vectors, each of which is vertical to the others, are created. These three vectors can then become the transformation matrix.

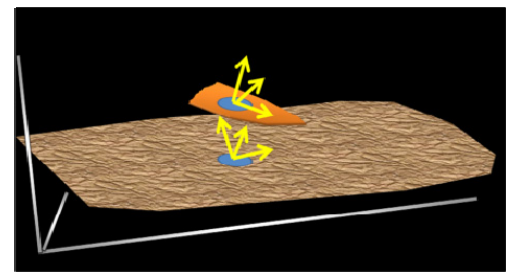

Fig. 6 Three axes.

Shown below is the transformation matrix.

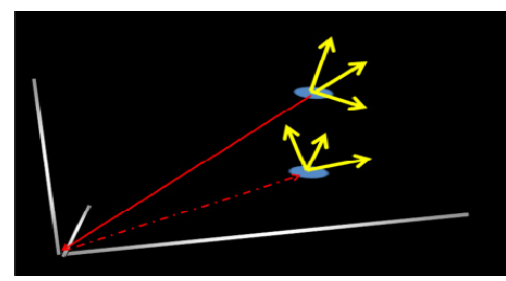

Fig. 7 Robotic inverse transformation.

Two transformation data, each of which results from two regions, can be calculated. Merging the local region with the global region requires inverse transformation.

As shown in the above equation, all the points in the local region are transformed once using local transformation, and are multiplied by the global map inversion of the global transformation matrix. In other words, by multiplying the inversion of the global transformation matrix to the local transformation matrix, the transformation matrix by which the $\mathrm{x}$ and $\mathrm{y}$ axes of the local region is parallel to the $\mathrm{x}$ and $\mathrm{y}$ axes of the global region can be calculated. 


\section{(c) Transformation-2}

After correcting the rotation of the two axes, place the local region at the accurate location by calculating the distance.

Determine the Euclidian distance among the center points of the planes, which were calculated at the previous stage, and perform translation.

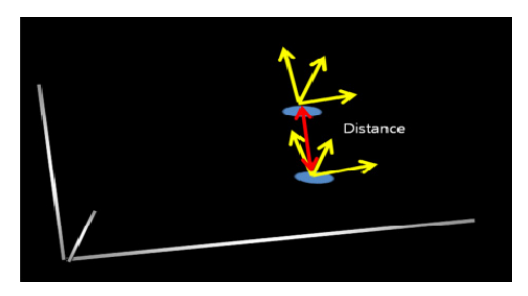

Fig. 8 Translation by the determined distance.

\section{(d) ICP}

The ICP algorithm is based on the principle that after translating the coordinates using the representative value of two datasets (e.g., the center of gravity) so that the error of the two representative values becomes minimal, such a translation (rigid-body fluctuation) is repeated until the error between the two datasets is within the determined error. Using ICP, the $\mathrm{z}$ rotation is adjusted.

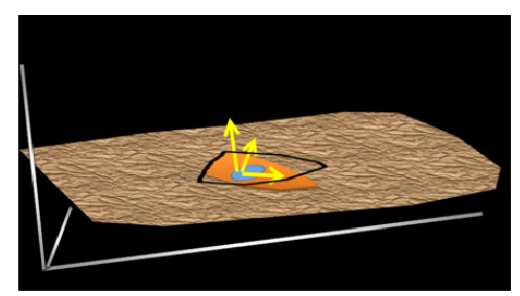

Fig. 9 Local and global regions by z rotation.

\section{RESULT}

The study tested the proposed algorithm by applying it to actual data, which are the point cloud information acquired from the site where an excavator is to be used. As global points are very large, some of them were excluded for easy calculation.

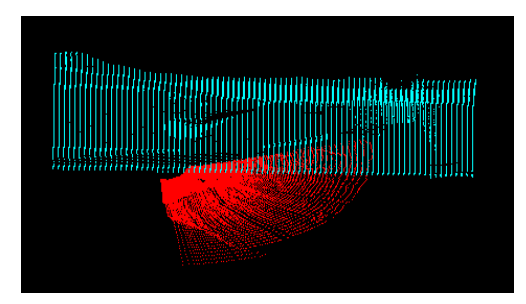

Fig. 10 The region taken from the global map and the local region.

Between the two regions exists a $20-\mathrm{cm}$ error between the $\mathrm{x}$ and $\mathrm{y}$ axes. That is, the local model is $20 \mathrm{~cm}$ on top of the global model. Moreover, the roll and pitch errors are within 20 degrees. The error on the $\mathrm{z}$ axis is within 60 degrees.

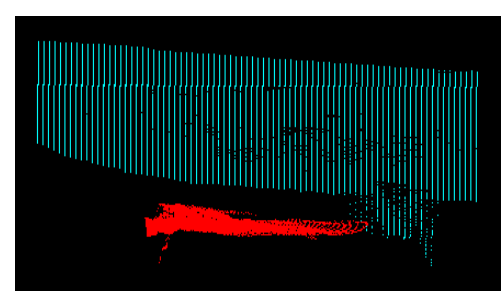

Fig. 11. The original error between the two models.

To make the two models parallel to each other, find the planes from the two models. These two planes, however, should have the same $\mathrm{x}$ and $\mathrm{y}$ values both in the global and local models. That is, they should be in the same place.

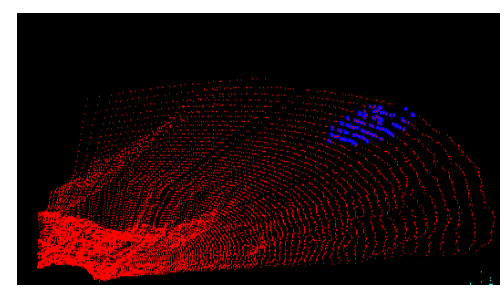

Fig. 12 Discovering a plane in the local model.

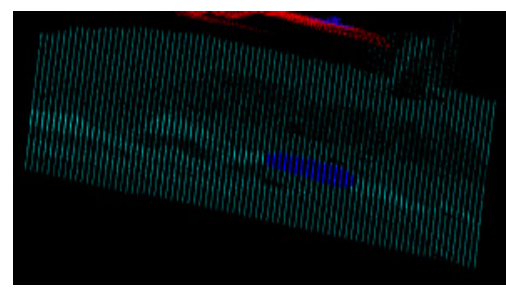

Fig. 13 Discovering a plane in the global model.

Once the planes are discovered in the two models, estimate the normal vector of the two planes, create two vectors centered on the normal vector, and rotate them. After the rotation, use ICP to adjust the $\mathrm{z}$ axis error. 


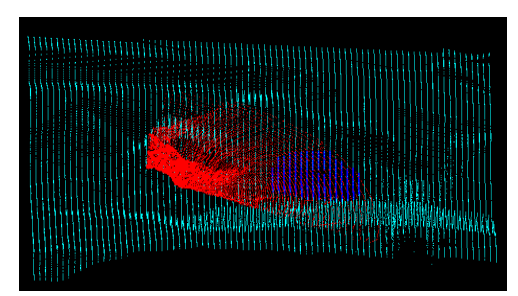

Fig. 14 Result of the merging of the two models.

The result shows that the merged model resulting from the proposed model has a considerably smaller error than that of the merged model based on the previous method, that is, creating the global model using the laser and then creating the local model. The translation by the $\mathrm{x}$ and $\mathrm{y}$ axes was within $10 \mathrm{~cm}$, the rotation of the local data by the ax and y axes was within 10 degrees, and the translation and rotation by the $\mathrm{z}$ axis were also reduced within $10 \mathrm{~cm}$ and 20 degrees, respectively. The proposed algorithm can merge any two models if planes can be found in the models.

\section{ACKNOWLEDGEMENT}

This work was supported by the Korean Institute of Construction \& Transportation Technology Evaluation and Planning (KICTEP) for the project program, "06-Unified and Advanced Construction Technology Program-C01.Intelligent Excavation System" funded by Ministry of Land, Transportation and Maritime Affairs(MLTM) of Korean government.

\section{REFERENCES}

[1] Besl P.J. and McKay H.D., "A method for the registration of 3D shapes," IEEE Trans. Pattern Anal. Mach. Intell, Vol. 14(2), pp. 239-256, 1992.

[2] Park S.Y., Choi S.I., Moon J.Y., Kim J., and Park Y.W., "Localization of an unmanned ground vehicle using 3D registration of laser range data and DSM," Applications of Computer Vision (WACV), pp. 1-6, 2009.

[3] Sander Oude Elberink and George Vosselman, "Building reconstruction by target-based graph matching on incomplete laser data: analysis and limitations," International Institute for GeoInformation Science and Earth Observation, Hengelosestraat 99, P.O. Box 6, 7500 AA Enschede, The Netherlands, Vol. 9(8), pp. 6101-6118, 2009.

[4] Kim C.W., "Human-assisted 3D spatial modeling using laser rangefinder for the automation of construction equipment," Journal of AIK, Vol. 22(1), pp. 147-154, 2006.

[5] Son H.J, Kim C.W., Yoo J.Y., Kim H.K., Han S.H., and Kim M.K., "Application of flash LADAR to 3D spatial information acquisition on the construction site: performance review," Conference Proceeding of KICEM, pp. 909-1004, 2007.

[6] Zitova B. and Flusser J., "Image registration methods: a survey," Image and Vision Computing, Vol. 21(11), pp. 977-1000, 2003.

[7] Jost T. and Hugli H., "A multiresolution ICP with heuristic closest-point search for fast and robust 3D registration of range images," Proceedings of the Fourth International Conference on $3 D$ Digital Imaging and Modeling, pp. 427-433, 2003.

[8] Tsin Y. and Kanade T., “A correlation-based approach to robust point set registration," Proceedings of ECCV'04, Springer LNCS 3023, pp. 558-569, 2004.

[9] Yoo H.S., Kim Y.S., and Han S.W., "Development of a noise elimination algorithm of stereo-vision image for 3D terrain modeling," Journal of KICEM, pp. 145-154, 2009.

[10] Akinci B., Boukamp F., Gordon C., Huber D., Lyons C., and Park K., "A formalism for the utilization of sensor systems and integrated project models for active construction quality control," Automation in Construction, Vol. 15(2), pp. 124-138, 2006.

[11] Kwon S.W., Bosche F., Kim C., Haas C., and Liapi K.A., "Fitting range data to primitives for rapid local 3D modeling using sparse range point clouds," Automation in Construction, pp. 67-81, 2004. 